\title{
TOOL SELECTION: LEARNING PICK-AND-PLACE OPERATIONS USING SMARTPHONE AR TECHNOLOGY
}

\author{
Adrian Tocu, Iuliana Beldiman, Alin Cruceat and Alexandru Butean \\ Lucian Blaga University of Sibiu, Faculty of Engineering, Connected Intelligence Research Center \\ 10, Victoriei Bd., Sibiu, 550024, România
}

\begin{abstract}
This paper presents an ongoing development process for an experimental application that aims to train an inexperienced user to operate a mobile device and receive Augmented Reality (AR) guidelines for basic pick-and-place operations. The purpose of the presented work is to offer a learning environment for placing multiple physical tools in specific places, as indicated by various guideline notes. The current phase represents the 9'th experiment from a series of others (24) that are conducted in order to identify the proper specifications, challenges and development estimations for a training station (room) built for manufacturing industry workers. The application is developed from a gamified perspective allowing users to explore 2 different games. According to the rules of each game, the user advances to the next level if he manages to place each object in a corresponding placeholder until all the spaces from a $2 \times 3$ grid are occupied correctly. The process is timed and the user sees a custom countdown timer alongside with guiding messages and feedback. While most of technical challenges were successfully surpassed, the interaction together with the performance measuring are currently going through an improvement process meant to identify and assess the educational advantages offered by this type of solutions.
\end{abstract}

\section{KEYWORDS}

Mobile Application, Augmented Reality, Human Computer Interaction, Gamification

\section{INTRODUCTION}

As a relatively recent enhancement added to the virtual reality (VR) technology evolution, AR brings a totally different level of interaction. The applicability of AR is focused on a variety of areas, starting from most common ones like gaming, assistive e-health, e-learning, to other more complex use-cases like military applications, smart city, industry and many others as presented in (Marr from Forbes, 2018). In order to bring the power of AR closer to the user and contribute to far-reaching scenarios, the most important factor is to combine AR with various other technologies that offer flexibility and mobility while allowing multiple ways of exploring AR visualizations as presented by (Aaltonen and Lehikoinen, 2006).

The current paper presents an ongoing progress of an experimental application that uses AR capabilities of modern smartphones. The application (currently in alpha version) requires a smartphone with an Android operating system and AR support in order to use the detection and display features of the device for educational and training purposes. The presented test scenario uses a gamified experience in order to guide the user to recognize and place correctly several common bricolage tools into specific indicated placeholders. The guided toolbox is part of a series of experiments that will be used in the development of a self-configuring and dynamic industrial training module to overcome the current industry workforce challenges. Every experiment has a sole purpose of identifying the challenges behind a specific type of user interaction. The current use-case aims to understand how fast human workers can execute easy indications received through an AR-enabled mobile device in order to correctly execute dynamic pick-and place instructions. 


\section{EXPERIMENTAL APPROACH}

\subsection{Scenario Description}

The scene setup configuration was inspired by the medical use-cases where there are several tools on different tables and the actors (doctors or nurses) are operating with them. Also, the mixed reality environment design has taken into consideration the conclusions described in (Antoniou et al, 2017).

In this particular use-case (tool selection), the user is directed to pick a correct tool from a tool library (tool table). All the objects from the tool library were scanned before with Vuforia Object Scanner and a database stores the Object Data. There is a direct one to one correspondence between the tool library (software) and the tool table (physical object). Once an object is upheld, it has to be placed in the indicated spot on the placeholder table. The placeholder table is a physical table that has a one to one direct correspondence with a digital AR matrix (2X3) so that the system can process real time object tracking operations.

All the user actions are sequential, pre-established and without completing a certain task, the user cannot proceed further in the process. The placeholder has 6 boxes, as displayed in Figure 1, each one of them represented by a colorful pattern, very different one from another in order to favor the precision of the solution at this stage.

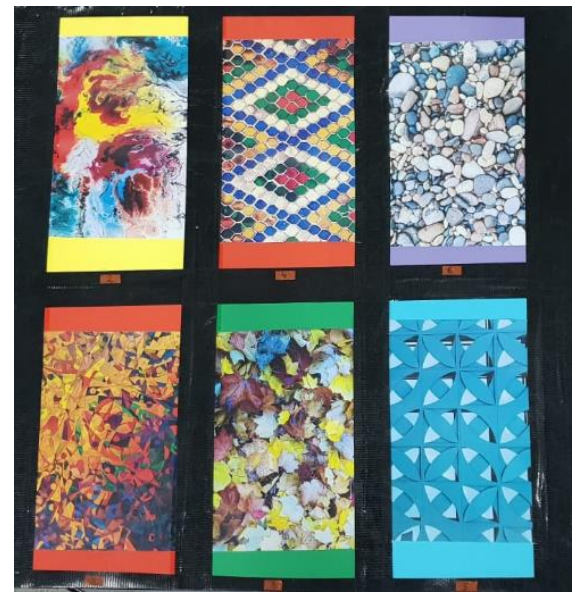

Figure 1. Placeholder table with 6 boxes as displayed on the working table

View from the top. Camera shot.

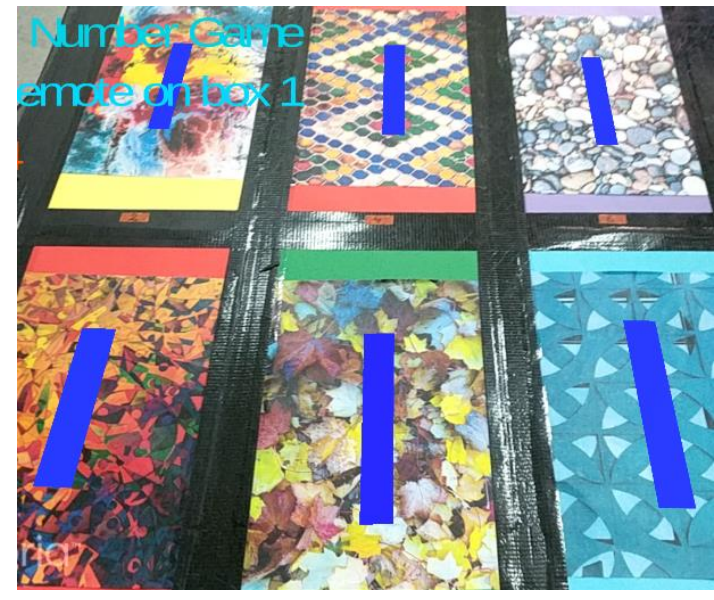

Figure 2. Placeholder table with 6 boxes and blue band strips for object placing direction.

View from the Android device screenshot

For guiding the correct tool placement, each box contains a colorful band strip (permanent AR object) that shows the correct direction for placing a tool. Without the proper tool direction, for some cases (marked with black stripes), even if the object would be placed in the right box the result would be considered incorrect. The color of the strip was chosen to be dark blue because of the big contrast with the background. The strip placement is affected by the distance and the tilting angle of the smartphone and it was one of the first signs of correct mapping, similar with the stabilizing methods described in (Andreasson, 2017). The empty placeholder with the blue direction strips are displayed in Figure 2.

\subsection{Gamified Experience}

In order to build a gamified experience, the whole process was designed around 2 available games:

- the game of numbers: the rule is to place an object in a placeholder that's indicated by a number (object $\mathrm{x}$ to box $\mathrm{n}$ mapping). The boxes are numbered in natural order.

- the game of colours: the rule is to place an object in a placeholder that's indicated by a color (object $\mathrm{x}$ to color $\mathrm{c}$ mapping). This game requires more attention since the placeholder images are very colorful, there is no order of the colors and multiple boxes can have the same color; 
For both games, each attempt is presented like a challenge against time with a countdown timer displayed directly from the beginning. In case a user doesn't put all the tools in the indicated boxes, after 120 seconds the game is interrupted. Every time a user makes a move, no mather if the move was correct or not, relevant instructions and feedback messages are displayed in order to guide him for the next step until he manages to place all the objects, as shown in Figures 3 and 4.

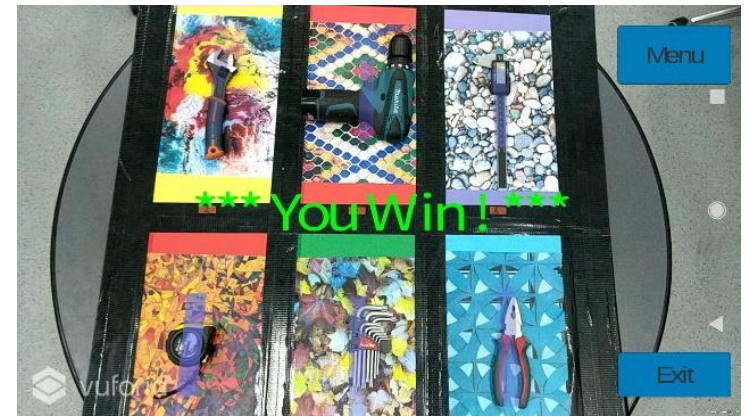

Figure 3. Completed board with all the 6 tools placed correctly and within the 120 seconds timeframe

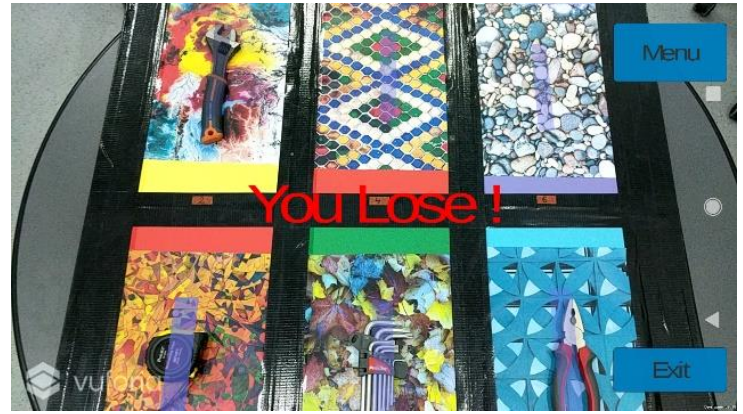

Figure 4. Incomplete board, the 120 seconds have passed and not all the tools are in the correct position

\subsection{Scenes}

Unity allows the creation of multi scene applications. Each scene can have its own isolated environment that can contain components of several types. A scene can contain basic elements like buttons, pop-up messages and virtual objects, or more complex ones like animations and manipulations. As described in (Kim et al, 2014), this particular feature allowed us to split the application in two scenes: START (only with basic elements) and GAME (mostly with complex elements).

The START scene as displayed in Figure 5, has elements from the interface that are visible on the phone's screen while running the application. In this scene a menu is displayed which has 3 native buttons and displays messages depending on what button you press. Through the menu the user can select what type of game they want to play.

The GAME scene, as displayed in Figure 6, represents the game's environment and has interface elements, buttons and text messages Vuforia-specific elements, that allows the use of virtual reality, AR Camera functions that operate with the scanned objects (Object Target) and the recorded images (Image Target).

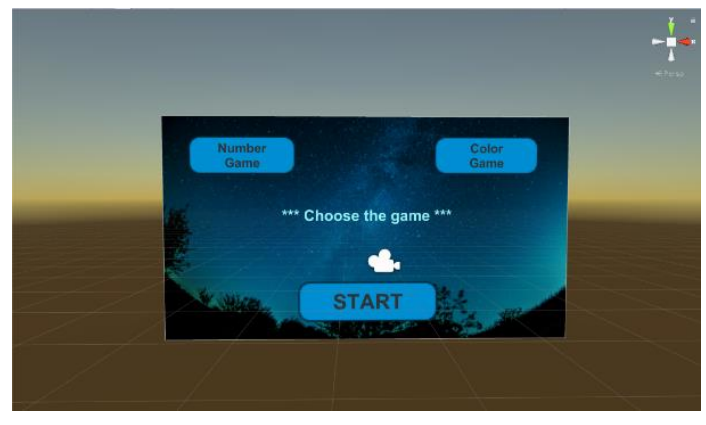

Figure 5. Start Scene as Displayed in the Unity View

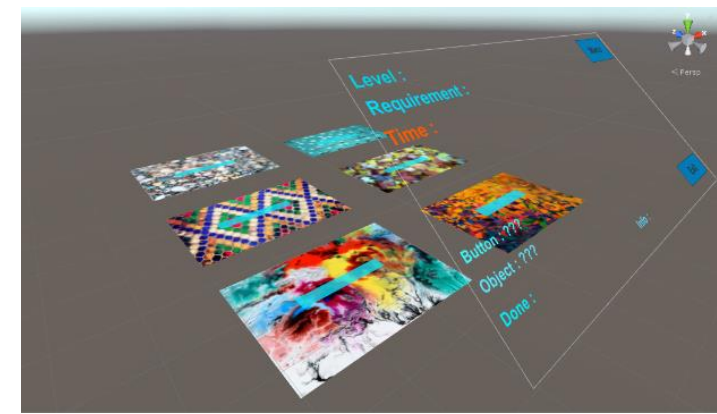

Figure 6. Game Scene as Displayed in the Unity View 


\section{EARLY PERFORMANCE ANALYSIS}

During the development process, the application performance was tested on several devices in different environments and their performance was rated below as displayed in Table 1.

Table 1. Performance Rating for Devices used during the Development Process

\begin{tabular}{|c|c|c|c|}
\hline Device Name & Camera Resolution (mp) & Operating System & Rating (1-5) \\
\hline LG Nexus 5X & 12 & Android 8.0 & 4 \\
\hline Samsung Galaxy J3 2016 & 8 & Android 5.1 & 3 \\
\hline ZTE Blade V8 & $13+2$ (dual) & Android: 7.0 & 5 \\
\hline Xiaomi Redmi Note 5 & $12+5$ (dual) & Android: 8.1 & 4 \\
\hline Google Pixel XL & 12 & Android 9.0 & 5 \\
\hline
\end{tabular}

The testing stage also concluded that there are 3 main factors that influence the overall performance:

- lens camera quality (not the resolution) - device feature - directly impacts detection speed;

- reflection of light - environment feature - multiple tests were conducted in several different rooms and when the artificial light was very strong the objects were glossy, and the detection precision decreased drastically;

- complexity of the item - object feature - at this stage, in order to increase the accuracy of scanned objects we decided to use only the items that have a large number of Vuforia Object Scanner Points (more than 100). A surface scan for a drilling machine is shown in Figure 7.

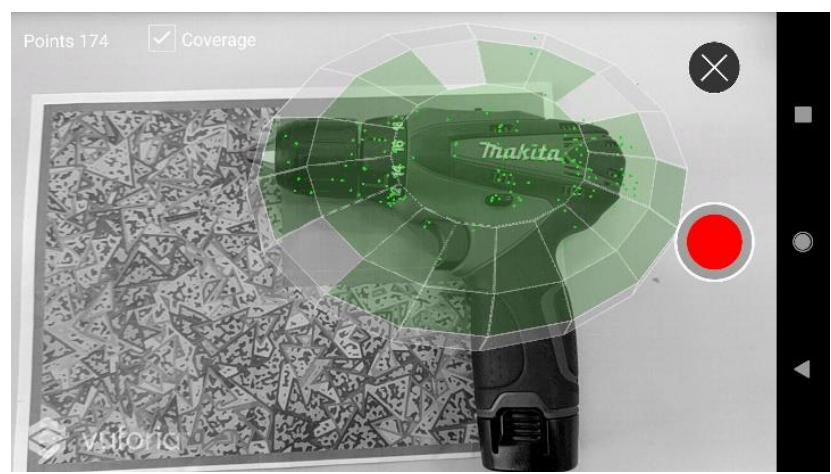

Figure 7. Surface of the Objects and Number of Vuforia Object Scanner Points for a Drilling Machine

For the testing stages of the alpha version, after the development was considered completed, it was decided to use the top 6 objects that had more scanner points (Adjustable Wrench, Allen Keys, Cordless Drill, Measuring Tape, Digital Caliper, Pliers), a laboratory with warm LED light (the natural light was shaded with translucent curtains) and the Google Pixel XL device (very good camera lens quality and superior Android version available).

\section{RESULTS AND CONCLUSION}

This paper presented an experimental application that follows an interactive method for learning pick-and-place operations using mobile AR technology. Intentionally, the operations were designed to be as simple as possible for the alpha version in order to focus on the following development tasks

- precise detection for smaller and medium size objects;

- game logic with custom difficulty countdown timer;

- validation of every step actions;

- basic user interface, messages and real time feedback;

- performance optimizations when the number of objects on the board was increasing. 
From a development perspective, the combination between Vuforia, Unity and Android represented a really challenging technical task, but under controlled light conditions and by using the proper mobile devices, the alpha version behaved unexpectedly good.

Up to this stage, the current experiment is considered to be successful and very relevant for the bigger purpose (training station progress). During the development stage and after the first alpha version was finalized, the application was tested by more than 20 people of different ages, but with the same technical background (computer science students, professors, researchers). All the subjects were able to complete both games in less than 5 attempts. Most of the problems and delays were related to the usability of the mobile phone with AR capabilities and once the users understood the technology philosophy everything became clear. This outcome shows that the users can be trained using the described method and probably they can easily adapt without difficulties to more complex scenarios developed under the same logic.

The overall performance of the application (object detection speed and precision) is highly influenced by the performance of the device and especially by the quality of the camera. The future extended study will also include detailed and explained performance measurements and recommendations for minimum system requirements, similar with the evaluation in (Fernandes, Cota, Moreira, 2015) and (Grahn, 2017).

Until now, most of the technical challenges were identified and solved. Despite that, there are still two issues that remain unsolved and without solutions:

- colorful scenes without human faces are considered complex by the internal Android autofocus feature and it would be very important to be able to control (force) the camera autofocus in order to speed up the placeholder detection

- detection of smaller objects using the zoom feature or by approaching the smartphone to the target area

The application is currently improved by adding the following changes and features:

- expand the grid to a more complex physical board $(6 * 3)$;

- detect multiple objects at the same time and ignore an object after it was correctly placed

- interchangeable images to allow various camera efficiency measurements;

- remote features as experimented in (Mourtzisa, Zogopoulosa and Vlachoua, 2017);

- gradual complexity levels to ensure a more natural learning process;

- multiplayer features for collaborative tasks;

- Microsoft Hololens AR glasses support, as experimented in (Heinonen, 2018).

\section{ACKNOWLEDGEMENT}

This work is supported through the DiFiCIL project (contract no. 69/08.09.2016, ID P_37_771, web: http://dificil.grants.ulbsibiu.ro), co-funded by ERDF through the Competitiveness Operational Programme 2014-2020.

\section{REFERENCES}

Aaltonen, A., Lehikoinen, J., 2006, Exploring Augmented Reality Visualizations. Proceedings of the working conference on Advanced visual interfaces, Venezia, Italy, pp. 453-456

Amin, D., Govilkar, S., 2015, Comparative Study of Augmented Reality Sdk's. International Journal on Computational Sciences \& Applications (IJCSA), Vol. 5, No.1, pp. 11-26.

Andreasson, K. J., 2017. Stabilizing Augmented Reality views through object detection. KTH Royal Institute of Technology, Available at https://pdfs.semanticscholar.org/dbb4/a711fc8be29c1eb42f582ca4b2a41b671d58.pdf [10 November 2018].

Antoniou, P.E., Dafli, E., Arfaras, G., Bamidis, P.D., 2017. Versatile mixed reality medical educational spaces; requirement analysis from expert users.Personal and Ubiquitous Computing, Vol. 21, Issue 6, pp. 1015-1024.

Fernandes, T.S., Cota, E., Moreira, A.F., 2014. Performance Evaluation of Android Applications: a Case Study. Brazilian Symposium on Computing Systems Engineering, Manaus, Brazil, pp. 79-84. 
Grahn, I., 2017. The Vuforia SDK and Unity3D Game Engine - Evaluating Performance on Android Devices. Linköping University, Available at https://liu.diva-portal.org/smash/get/diva2:1127482/FULLTEXT01.pdf [10 November 2018].

Heinonen, E., 2018. HoloLens research and demo application development. Metropolia University of Applied Sciences, Available at https://www.theseus.fi/bitstream/handle/10024/142176/Heinonen_Eemeli.pdf [10 November 2018].

Huang, L., Gui, B., 2015. Research on the Application of Products based on Unity3D. International Symposium on Computers \& Informatics (ISCI 2015), Vol. 13, pp. 1213-1217.

Kim, S.L., Suk, H.J, Kang J.H., Jung J.M., 2014, Using Unity 3D to Facilitate Mobile Augmented Reality Game Development. IEEE World Forum on Internet of Things (WF-IoT), DOI: 10.1109/WF-IoT.2014.6803110.

Marr B., 2018. 9 Powerful Real-World Applications Of Augmented Reality (AR) Today. Forbes Magazine. Available at https://www.forbes.com/sites/bernardmarr/2018/07/30/9-powerful-real-world-applications-of-augmented-reality-artoday/\#2bfb92f2fe95 [10 November 2018].

Mourtzisa, D., Zogopoulosa, V., Vlachoua, E., 2017. Augmented reality application to support remote maintenance as a service in the Robotics industry. Procedia CIRP, Vol. 63, pp. 46-51.

Patil, P.P., Alvares, R., 2015, Best practices for cross-platform virtual reality development. International Journal of Advance Research in Computer Science and Management Studies,Vol. 3, Issue 4, pp. 19-27. 